\title{
Insumos utilizados en la preparación de alimentos en producción porcina y su potencial de contaminación por dioxinas en la carne
}

\author{
Feed materials used in the preparation of food in pork production \\ and its potential for dioxin contamination in the meat \\ F Samsing ${ }^{a}$, C Bustos-López ${ }^{b}$, JT Schoffera ${ }^{\mathrm{a}}$ CA Mattar ${ }^{\mathrm{ac}}$, A González $^{\mathrm{a}}$, \\ C Robles ${ }^{\mathrm{a}}$, O Acevedo ${ }^{\mathrm{a}}$ y CE Valdovinos ${ }^{\mathrm{a} *}$ \\ ${ }^{a}$ Centro de Investigaciones Ecotoxicológicas, Facultad de Ciencias Silvoagropecuarias, Universidad Mayor, Santiago, Chile. \\ ${ }^{\text {b} F a c u l t a d ~ d e ~ C i e n c i a s, ~ U n i v e r s i d a d ~ S a n t o ~ T o m a ́ s, ~ S a n t i a g o, ~ C h i l e . ~}$ \\ 'Programa de Doctorado en Ciencias Silvoagropecuarias y Veterinarias, Universidad de Chile, Santiago, Chile.
}

\begin{abstract}
SUMMARY
This study assessed the contribution of various feed ingredients used in swine feeding as a source of dioxins, furans and dioxin like polychlorinated biphenyls (DL-PCBs) contamination in pork, considering the dietary changes during breeding, raising and fattening. Raw materials or feed ingredients were separated into different categories, developing a dataset with EROD/H4IIE bioassay results (determination of 7-Ethoxyresorufin $O$-Deethylase activity in H4IIE hepatoma cell line). Two types of diets were established that considered the varying percentages of ingredients necessary during the productive cycle of these animals. These two diets were based on those of common use in Chile. A descriptive analysis of the information contained in the dataset was performed, characterising the observations behaviour. A transfer model in which the body burden of dioxin increases proportionally to the consumption of contaminated food was proposed. The highest average contaminant concentration, expressed as 2,3,7,8-tetrachlorodibenzo- $p$-dioxin equivalent toxics derived from the EROD/H4IIE bioassay (TCDD-EQ/g) was found in ingredients of mineral origin (16.21 pg TCDD-EQ/g), followed by those of fatty acid mixtures $(2.03 \mathrm{pg}$ TCDD-EQ/g), while the lowest average concentrations were found in dietary premixes $(0.29 \mathrm{pg}$ TCDD-EQ/g) and vegetable oils $(0.35 \mathrm{pg}$ TCDD-EQ/g). With regard to the fransfer model, the evaluation of the contribution of the different feed ingredients to the total amount of diet contamination showed that the vegetable components had the highest value, due to the high proportion of them in feed. The second highest contributor was the raw material of mineral source.
\end{abstract}

Palabras clave: compuestos orgánicos persistentes, contaminantes en carnes, químicos liposolubles, xenobióticos.

Key words: persistent organic pollutants, pollutants in meats, lipid-soluble chemicals, xenobiotics.

\section{INTRODUCCIÓN}

Las dibenzo-p-dioxinas policloradas (PCDDs), los dibenzofuranos policlorados (PCDFs) y los bifenilos policlorados similares a dioxinas (DL-PCBs) son compuestos orgánicos persistentes que se forman como subproductos en procesos industriales y que, mediante procesos de bioacumulación y biomagnificación, llegan a encontrarse en los alimentos. Es por ello que la alimentación es la principal vía a través de la cual los seres humanos sufren exposición a dichos compuestos (Svenson y col 1991). Los principales casos descritos de contaminación por dioxinas señalan que ésta se puede producir a diferentes niveles de la cadena alimentaria, siendo las materias primas contaminadas una de las principales vías de ingreso. Tal es el caso del descubrimiento en Estados Unidos y Europa de una alta contaminación por estos xenobióticos contenidos en arcillas caolínicas, y que fueron incorporadas a las dietas para peces y aves de corral (European Commission 2000, Schecter y col 2006).

Aceptado: 12.08.2011.

* Casilla 235 correo 34, Santiago, Chile; carlos.valdovinos@umayor.cl
Similarmente, se detectó contaminación por dioxinas en pellets de pulpa cítrica de Brasil y en grasa reciclada en Bélgica (Malisch 2000). Por otra parte, Corea detectó, en julio de 2008, la presencia de dioxinas en carne de cerdo chilena identificándose al óxido de zinc en una premezcla de minerales utilizada en las raciones como causa del incidente (Valdovinos, 2009). Estos hallazgos motivan a formular la pregunta de si ciertos insumos utilizados en las raciones presentan un mayor riesgo de contaminar la carne como producto final. Por lo tanto, el objetivo del presente estudio es evaluar la contribución de los diversos insumos utilizados en la alimentación de los cerdos, como fuente de contaminación de la carne, considerando las variaciones dietarias durante la recría, crianza y engorda.

\section{MATERIAL Y MÉTODOS}

\section{ELABORACIÓN DE BASES DE DATOS}

Según Valdovinos (2009) y Samsing (2010), las fuentes de contaminación de los animales de producción y en especial de los animales de granja, de acuerdo a la legislación de la Unión Europea (2006) y considerando la 
realidad chilena, podrían afectar a las siguientes categorías de materias primas o insumos que conforman las raciones de cerdos: materias primas de origen vegetal (excepto aceites), aceites vegetales, materias primas de origen mineral, ácidos grasos de origen animal, harinas de origen animal, harinas y aceites de pescado, aglutinantes y antiaglomerantes, productos lácteos y premezclas (complejos vitamínicos, aminoácidos, antibióticos y otros). Además de los insumos mencionados se agregó la categoría "mezcla de ácidos grasos", que en Chile se suele incorporar en la dieta como suplemento energético.

Para esto se consideró un conjuntos de datos ("EROD/ H4IIE') que incluye los insumos utilizados en la alimentación de cerdos, ordenados por categorías, y los resultados de los análisis de PCDDs, PCDFs y DL-PCBs, expresados en pg

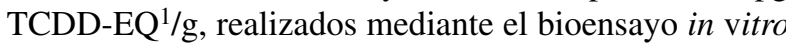
con la línea celular H4IIE, en el Centro de Investigaciones Ecotoxicológicas de la Universidad Mayor (CIEMAYOR) entre los años 2008 y 2010. Para aplicar el bioensayo se consideró el protocolo modificado de Tillitt y col (1991), el cual utiliza la línea celular de hepatoma de Rattus norvegicus, importada de la American Type Culture Collection (ATCC@, CRL-1548 ${ }^{\mathrm{TM}}$ ). La línea celular fue cultivada en un Medio de Eagle modificado por Dulbecco (D-MEM), enriquecido con un $15 \%$ de suero fetal bovino (SFB). Las células fueron mantenidas bajo condiciones estándares $\left(37{ }^{\circ} \mathrm{C}, 5 \% \mathrm{CO}_{2}\right)$ y se les permitió crecer entre 4 a 5 días, luego fueron sembradas en placas de microtítulo de 96 pocillos de fondo plano, Nunc ${ }^{\circledR}$, en un volumen de 300 $\mu \mathrm{L} /$ pocillo con una densidad de $1,2 \times 10^{4}$ células/pocillo. Después de 24 horas de crecimiento se procedió a dosificar el estándar de 2,3,7,8-tetraclorodibenzo- $p$-dioxina (TCDD) en un set de 7 diluciones seriadas en razón 1:2 (50 pg/pocillo a 0,069 pg/pocillo) con tres réplicas cada una (dilución 1:2). Dicho estándar fue usado para generar una curva de dosis respuesta con la cual todas las muestras fueron relacionadas.

Los extractos de las muestras y los controles de calidad se dosificaron en un set de 7 en razón 1:3, con 3 réplicas cada una. Luego de la dosificación se dejó a las células bajo condiciones estándares por 72 horas para su posterior lectura. Para ello el medio fue removido a través de un lavador de placas Thermo ${ }^{\circledR}$ Weelwash $4 \mathrm{Mk}$ 2 , que utiliza agua ultrapura, dejando aproximadamente $60 \mu \mathrm{L}$ de medio/pocillo. Terminado el lavado se incuban las células por 5 minutos, lo que produce citólisis osmótica, dando como resultado la exposición del citocromo P-4501A1 (CYP1A1). Pasado el tiempo se retiró la placa de la incubadora y se le agregaron $20 \mu \mathrm{L}$ de buffer a $37^{\circ} \mathrm{C}$ con $80 \mu \mathrm{L}$ de dicumarol. Posteriormente, se adicionaron $20 \mu \mathrm{L}$ de $5 \mu \mathrm{M}$ de etoxiresorufina y $20 \mu \mathrm{L}$ de $5 \mu \mathrm{M}$ de NADPH. Finalmente, se colocaron las placas en un lector de fluorescencia (BioTek ${ }^{\circledR}$ FLx800 8 TM $)$, que realiza lecturas

En este documento se utilizará TCDD-EQs para denominar los tóxicos equivalentes (TEQs) derivados del bioensayo EROD-H4IIE. por 20 minutos con un filtro de excitación $530 \mathrm{~nm}$ y con un filtro de emisión de $580 \mathrm{~nm}$. Este método permite la lectura de la actividad EROD así como también la lectura de la proteína en el mismo pocillo (Kennedy y Jones 1994). En la etapa final del ensayo se utilizaron curvas de dosis respuestas para determinar la potencia relativa del extracto (RPFs) comparando los valores de las pendientes de los extractos de las muestras con los valores de la pendiente del estándar de TCDD para obtener los equivalentes tóxicos (TCDD-EQs) expresados en pg/g de muestra (Mason y col 1985, Tillitt y col 1993, Whyte y col 2004).

Mediante entrevistas a médicos veterinarios a cargo del área de sanidad y producción de dos empresas, que en conjunto generan el $70 \%$ de la carne de cerdo en Chile ${ }^{2}$, se establecieron dos dietas de referencia utilizadas en el país (D1 y D2, cuadro 1), las cuales consideraron los porcentajes de incorporación de materias primas, que varían a lo largo del ciclo productivo del cerdo (crianza, recría y engorda).

\section{ANÁLISIS ESTADÍSTICO}

Se realizó un análisis descriptivo de la información contenida en el conjunto de datos, utilizando el software Minitab® versión 15.1 para Windows (Minitab Inc, State College, PA, USA), caracterizando el comportamiento de las observaciones y se propuso un modelo de transferencia.

\section{CONTRIBUCIÓN DE LOS INSUMOS A LA CONTAMINACIÓN} FINAL

Se tomaron los promedios de contaminación para cada insumo del conjunto de datos (cuadro 2), para obtener la contribución promedio porcentual en la dieta de crianza, recría y engorda (cuadro 1), en pg TCDD-EQ/g para D1 y D2. Finalmente, se llevó a porcentaje la contribución de los insumos a la contaminación final en todas las dietas.

Modelo de transferencia de PCDDs, PCDFs y DLPCBs a la carne de cerdo.

A fin de predecir los niveles de PCDDs, PCDFs y DL-PCBs en el tejido, Fries (1996) propone un modelo de transferencia desde la dieta al producto final, dado por la siguiente expresión:

$$
C_{t}=B_{t} / F_{t} \text {, si } t>0 \text {. }
$$

Donde $C_{t}$ es la concentración de dioxinas al tiempo $t, B_{t}$ es la carga corporal de dioxinas al tiempo $t$ y $F_{t}$ es la grasa corporal al tiempo $t$. Según Fries (1995), el modelo tiene como supuesto inicial que las PCDDs, PCDFs y DL-PCBs se distribuyen en concentraciones relativamente uniformes en el tejido graso. Además, se considera que la captación de estos xenobióticos es un proceso pasivo y la eliminación,

\footnotetext{
http://www.asprocer.cl Fecha de consulta: 09 de junio de 2011.
} 
Cuadro 1. Dieta de referencia usada en Chile en producción porcina (D1 y D2).

Reference diet used in Chile in pork production (D1 \& D2).

\begin{tabular}{|c|c|c|c|c|c|c|c|}
\hline \multirow{3}{*}{\multicolumn{2}{|c|}{ Insumos }} & \multirow{2}{*}{\multicolumn{2}{|c|}{$\frac{\text { Recría }}{\text { (21-70 días) }}$}} & \multirow{2}{*}{\multicolumn{2}{|c|}{$\frac{\text { Crianza }}{\text { (71-120 días) }}$}} & \multirow{2}{*}{\multicolumn{2}{|c|}{$\frac{\text { Engorda }}{\text { (121-180 días) }}$}} \\
\hline & & & & & & & \\
\hline & & D1 & D2 & D1 & D2 & D1 & D2 \\
\hline \multicolumn{2}{|c|}{ Materias primas de origen vegetal } & 61,17 & 61,17 & 92,02 & 91,05 & 92,74 & 91,05 \\
\hline \multicolumn{2}{|c|}{ Harina de pescado } & 10,50 & 10,50 & - & - & - & - \\
\hline \multicolumn{2}{|c|}{ Materias primas de origen mineral } & 2,25 & 2,25 & 1,69 & 2,15 & 1,73 & 2,15 \\
\hline \multirow[t]{3}{*}{ Ácidos grasos } & Mezclas (a) & - & - & 3,50 & - & 2,75 & - \\
\hline & Origen animal & - & - & - & 2,10 & - & 2,10 \\
\hline & Aceite vegetal & - & - & - & 4,05 & - & 4,05 \\
\hline \multicolumn{2}{|c|}{ Harinas de origen animal (b) } & - & - & 1,75 & - & 1,75 & - \\
\hline \multicolumn{2}{|c|}{ Aglomerantes y antiaglutinantes } & - & - & 0,21 & - & 1,21 & - \\
\hline \multicolumn{2}{|c|}{ Próductos lácteos } & 25 & 25 & - & - & - & - \\
\hline \multicolumn{2}{|l|}{ Premezclas(c) } & 1,08 & 1,08 & 0,83 & 0,65 & 0,82 & 0,65 \\
\hline
\end{tabular}

(a) Mezclas de ácidos grasos de origen animal, vegetal y aceites reciclados (e.g. de frituras).

(b) Harinas de carne y hueso, de sangre, de plumas y otros subproductos de animales terrestres.

(c) Incluye enzimas, complejos vitamínicos, aminoácidos, antibióticos y otros aditivos alimenticios.

según Fries (1996) y Huwe (2006), es despreciable durante la vida del cerdo con tasas de $0,005 \%$ (200 días de vida media) a $0,01 \%$ (100 días de vida media).

Luego, Huwe (2006) propone:

$$
F_{t}=0,0028 \cdot t^{1,8173} \text {, si } t>0 \text {. }
$$

Por consiguiente, el presente trabajo propone un modelo multiplicativo para determinar $B_{t .}$ a través de una adaptación del modelo de Fries (1996), en que la carga corporal de dioxinas aumenta en forma proporcional al consumo de alimentos contaminados. El modelo propuesto es el siguiente:

$$
B_{t}=A \cdot I \cdot D \cdot C A \cdot t, \text { si } t>0 .
$$

Donde $A$ es el coeficiente de absorción, $I$ es el porcentaje del insumo incluido en la dieta, $D$ es la contaminación promedio del insumo, $C A$ es el consumo diario de alimento y $t$ los días de exposición.

Se determinó mediante (3) la carga corporal de dioxinas en cada etapa de crecimiento del animal, puesto que los valores de $I$ y $C A$ varían en el ciclo productivo. Aunque diversos autores afirman que la absorción de estos xenobióticos, a través de la dieta es de aproximadamente un $20 \%$ (Iben y col 2003, Huwe 2006, Brambilla y col 2008), se proponen porcentajes de absorción para $A$, que fluctúan entre $5 \%$ y $100 \%$. Los valores de $I$ se obtuvieron de las dietas de referencia utilizadas en Chile, $D$ se obtuvo del análisis descriptivo del conjunto de datos "EROD/H4IIE".
Bajo condiciones de alimentación ad libitum, el consumo diario de alimento está en función del peso corporal $(P)$ y se obtiene de la siguiente ecuación (Fries 1996, Huwe 2006):

$$
C A_{P}=4,003\left(1-\mathrm{e}^{-0,01768 \cdot P}\right)
$$

Para el cálculo de la carga corporal de cada etapa se utiliza $C A$, promedio del consumo al inicio y al final de la etapa, $P$ (en $\mathrm{kg}$ ) se obtiene de la propuesta de Huwe (2006):

$$
P_{t}=0,7716 \cdot t-2,9701 \text {, si } t>0 \text {. }
$$

\section{RESULTADOS}

Las dietas D1 y D2 varían en los porcentajes de insumos incorporados en la crianza y engorda, mientras que en la recría la composición de la dieta es la misma. A su vez, D1 utiliza como fuente energética las mezclas de ácidos grasos, mientras que D2 incluye ácidos grasos de origen animal y aceites vegetales.

Del conjunto de datos "EROD/H4IIE" (cuadro 2), la media de contaminación más alta corresponde a las materias primas de origen mineral, con 16,21 pg TCDD-EQ/g (valor máximo de 429,15 pg TCDD-EQ/g), seguido por las mezclas de ácidos grasos (2,03 pg TCDD-EQ/g). Las concentraciones medias más bajas de contaminación se presentan en las premezclas $(0,29 \mathrm{pg}$ TCDD-EQ/g) y los aceites vegetales $(0,35 \mathrm{pg}$ TCDD-EQ/g). 


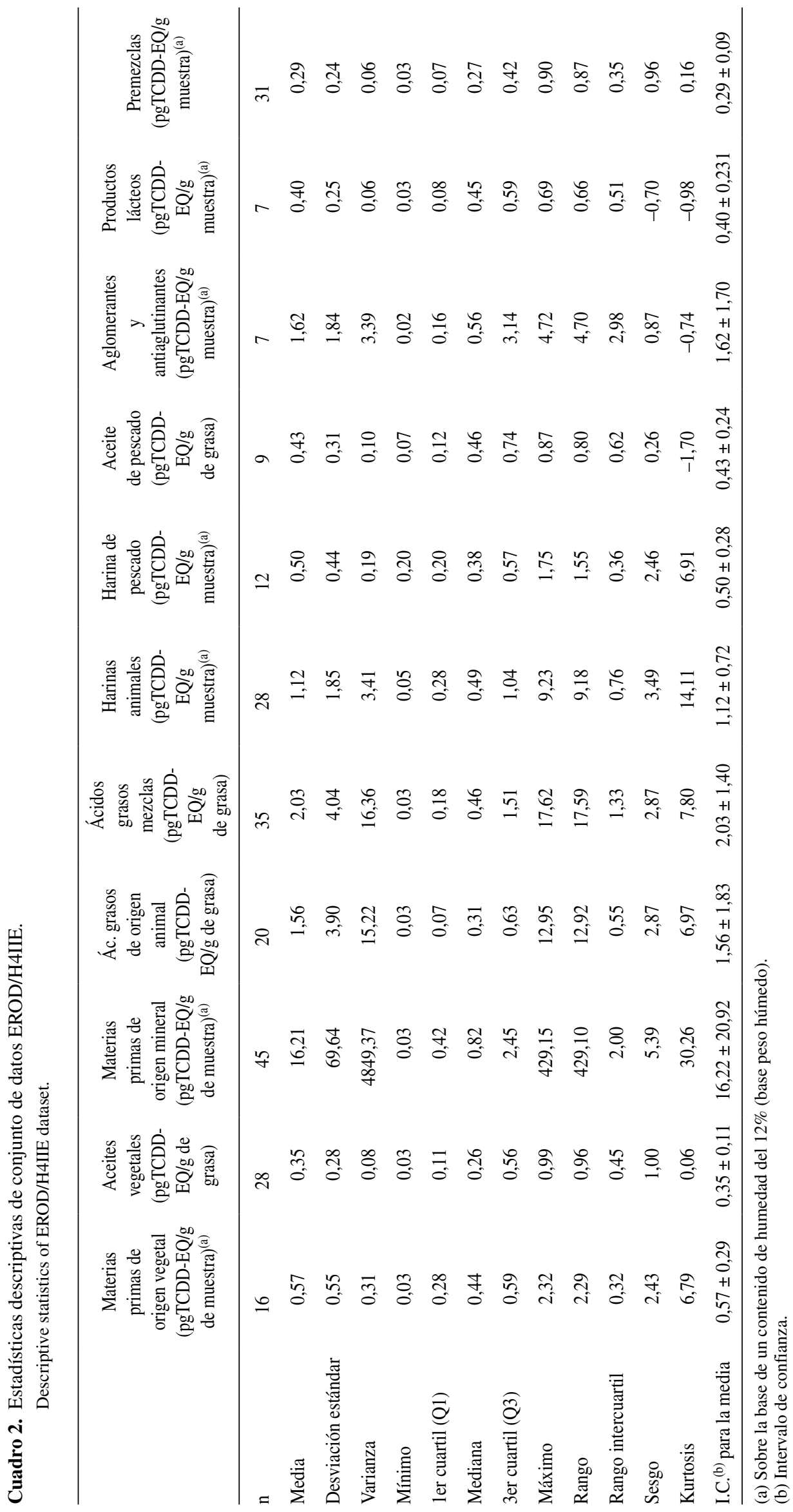


Cuadro 3. Concentraciones de PCDDs, PCDFs y DL-PCBs $(\mathrm{Ct})$ en la carne de cerdo al término de cada etapa del ciclo productivo, usando D1 y considerando como fuente de contaminación las materias primas de origen vegetal (media= 0,57 pg TCDD/g de muestra, conjunto de datos "EROD/H4IIE") y ácidos grasos (promedio contaminación = 2,03 pg TCDD/g de muestra, conjunto de datos "EROD/ H4IIE").

PCDDs, PCDFs and DL-PCBs concentrations $(\mathrm{Ct})$ in pork at the end of each stage of production cycle, using D1 and considering the vegetal origin raw materials (mean $=0.57 \mathrm{pg}$ TCDD/g sample, "EROD/H4IIE" dataset) and fatty acid (mean $=2.03 \mathrm{pg}$ TCDD/g sample, "EROD/H4IIE" dataset) as contamination source.

\begin{tabular}{|c|c|c|c|c|c|c|c|c|}
\hline \multirow{3}{*}{ Absorción } & \multicolumn{8}{|c|}{ Concentración (pg TCDD-EQ/g de grasa) } \\
\hline & \multicolumn{2}{|c|}{$\mathrm{C}_{70}$} & \multicolumn{2}{|c|}{$\mathrm{C}_{120}$} & \multicolumn{2}{|c|}{$\mathrm{C}_{180}{ }^{(\mathrm{a})}$} & \multicolumn{2}{|c|}{$\mathrm{C}_{220}$} \\
\hline & $\mathrm{MPOV}^{(\mathrm{b})}$ & $A G^{(c)}$ & $\mathrm{MPOV}^{(\mathrm{b})}$ & $A G^{(c)}$ & $\mathrm{MPOV}^{(\mathrm{b})}$ & $A G^{(c)}$ & $\mathrm{MPOV}^{(\mathrm{b})}$ & $A G^{(c)}$ \\
\hline $100 \%$ & 4,42 & 0,00 & 6,00 & 0,59 & 5,94 & 0,61 & 5,68 & 0,59 \\
\hline $80 \%$ & 3,54 & 0,00 & 4,48 & 0,47 & 4,76 & 0,49 & 4,54 & 0,47 \\
\hline $60 \%$ & 2,65 & 0,00 & 3,60 & 0,35 & 3,57 & 0,36 & 3,41 & 0,35 \\
\hline $40 \%$ & 1,77 & 0,00 & 2,40 & 0,24 & 2,38 & 0,24 & 2,27 & 0,23 \\
\hline $20 \%$ & 0,88 & 0,00 & 1,20 & 0,12 & 1,19 & 0,12 & 1,14 & 0,12 \\
\hline $10 \%$ & 0,44 & 0,00 & 0,60 & 0,06 & 0,59 & 0,06 & 0,57 & 0,06 \\
\hline $5 \%$ & 0,22 & 0,00 & 0,30 & 0,03 & 0,30 & 0,03 & 0,28 & 0,03 \\
\hline
\end{tabular}

(a) Concentración de PCDDs, PCDFs y DL-PCBs al momento de la faena (180 días).

(b) Materias primas de origen vegetal.

(c) Ácidos grasos.

\section{RESULTADOS DEL MODELO PROPUESTO}

Considerando distintos porcentajes de absorción de dioxinas $(100 \%, 80 \%, 60 \%, 40 \%, 20 \%, 10 \%$ y $5 \%)$ en (4) se obtiene la contaminación estimada de la carne (en pg TCDD-EQ/g de grasa) al final de cada etapa del ciclo productivo (final de recría o $C_{70}$; final de crianza o $C_{120}$; final de engorda o $C_{180}$ y final de postengorda o $C_{220}$ ), para cada una de las dietas. El cuadro 3 muestra las concentraciones estimadas de PCDDs, PCDFs y DL-PCBs en la carne de cerdo, para la dieta D1 considerando las materias primas de origen vegetal y los ácidos grasos como fuente de contaminación, las cuales presentan una media de 0,57 y 1,56 pg TCDD-EQ/g respectivamente (cuadro 2). Por otra parte, la dieta D1 no considera el aporte de mezclas de ácidos grasos, por tanto, la carga corporal al final de esta etapa es cero $\left(C_{70}=0\right)$. Los resultados de la estimación aumentan hasta alcanzar cierto nivel y disminuyen después de los 120 días (figura 1A). Para una absorción del 20\%, la estimación de la concentración más alta es de 1,20 pg TCDD-EQ/g de grasa a los 120 días, en cambio a los 180 días la concentración estimada es de 1,19 pg TCDD-EQ/g de grasa. Si la faena es a los 220 días, la concentración se estima que disminuye a 1,14 pg TCDD-EQ/g de grasa. En
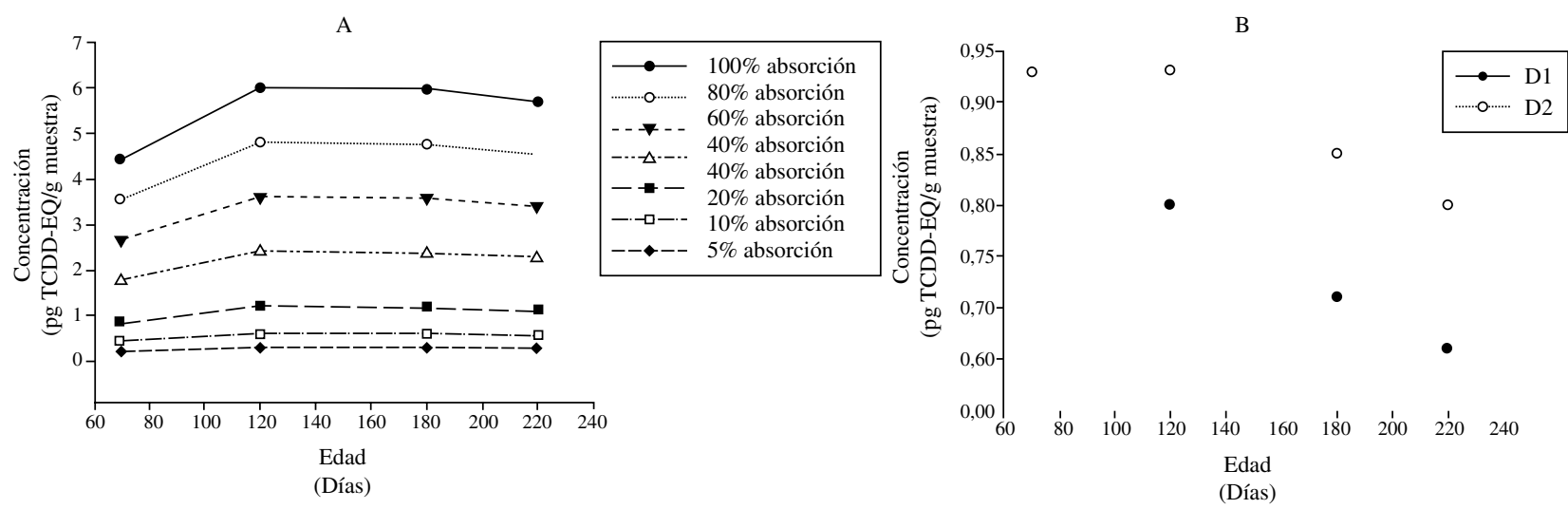

Figura 1. (A) Concentraciones de PCDDs, PCDFs y DL-PCBs en la carne de cerdo, considerando las materias primas de origen vegetal como fuente de contaminación (media $=0,57 \mathrm{pg}$ TCDD-EQ/g de muestra, conjunto de datos "EROD/H4IIE") administrando D1 (dieta1). (B) Concentraciones de PCDDs, PCDFs y DL-PCBs en la carne de cerdo, considerando como fuente de contaminación las materias primas de origen mineral (media = 16,21 pgTCDD-EQ/g de muestra, conjunto de datos "EROD/H4IIE") con un 20\% de absorción, en D1 (dieta1) y D2 (dieta 2).

(A) PCDDs, PCDFs and DL-PCBs concentrations in pork considering the vegetal origin raw materials as a contamination source $($ mean $=0.57 \mathrm{pg}$ TCDD-EQ/g sample, "EROD/H4IIE" dataset) administering D1 (diet1). (B) PCDDs, PCDFs and DL-PCBs concentrations in pork considering the mineral origin raw materials as a contamination source (mean $=16.21 \mathrm{pg}$ TCDD-EQ/g sample "EROD/H4IIE" dataset) with a $20 \%$ absorption in D1 (diet 1) and D2 (diet 2). 
la figura 1B se observa que D1 y D2 difieren levemente en el comportamiento de la concentración estimada en el tejido para el insumo de origen mineral.

\section{DISCUSIÓN}

Diversos esfuerzos se han realizado para estimar las concentraciones de PCDDs, PCDFs y DL-PCBs en carne de cerdo, aves de corral y carne de vacuno, en relación con la exposición a través de los insumos de la dieta, los cuales utilizan los resultados de los análisis realizados mediante HRGC/HRMS (Fries 1996, Guruge y col 2005, Van Eijkeren y col 2006, Brambilla y col 2008). En cambio, en el presente trabajo los insumos de las dietas de los cerdos fueron analizados mediante el bioensayo EROD/ H4IIE, utilizado en Chile como método de screening o cribado para la detección de PCDDs y PCDFs en carnes de pollos broiler (Schoffer y col 2011). Dicho bioensayo se caracteriza por su rapidez y confiabilidad en la entrega de resultados y menor costo, frente al método cromatográfico (Whyte y col 2004, Schoffer y col 2011) y puede ser utilizado en programas de monitoreo de insumos de alimentos para animales.

Los modelos desarrollados para estimar la contaminación en la carne deben considerar diferentes variables, tales como perfil de congéneres, características fisicoquímicas y toxicocinéticas, tiempo de exposición, genética, estado fisiológico de los animales, régimen de alimentación y otras (Hoogenboom y col 2004, Brambilla y col 2008). El modelo propuesto incorporó la mayoría de las variables mencionadas, mediante la aplicación de fórmulas de crecimiento y aumento de la masa corporal. La variable perfil de congéneres no fue considerada, ya que el resultado del bioensayo EROD/ H4IIE entrega un resultado total o valor de equivalencia total (TEQ), en que no se diferencian los congéneres. Con respecto a las propiedades toxicoquinéticas de PCDDs y PCDFs, Pirard y De Pauw en 2005 demostraron que no existen diferencias significativas en la absorción de ambos grupos. Una vez absorbidos por el organismo, la metabolización de estos compuestos es muy baja y, por tanto, su excreción es casi despreciable (Fries 1996, Hoogenboom y col 2004, Spitaler y col 2004), observándose una prolongada vida media de las dioxinas en seres humanos, que oscila entre 7 y 11 años (Pirkle y col 1989).

La absorción de PCDDs y PCDFs también va a depender de la matriz a la cual se encuentran asociados, afectando su biodisponibilidad. Al respecto, la absorción desde matrices lipídicas (aceites y ácidos grasos) sería mayor (Fries y Marrow 1975, Jones y col 1989), por tanto, estos insumos contaminados constitutirían un riesgo mayor.

El modelo propuesto por Huwe (2006) utiliza fórmulas de crecimiento y de aumento de la grasa corporal obtenidas de un estudio realizado en cerdos de engorda de raza mixta en el Biosciences Research Laboratory del Agricultural Research Service (ARS), que depende del Departamento de Agricultura de los Estados Unidos (USDA). Es posible que las razas de cerdo criadas en Chile no presenten la misma tasa de crecimiento, por lo que el modelo podría ser más preciso, si se ajustan estas fórmulas a la realidad de Chile e incluso a la de cada plantel. Las razas de cerdo han cambiado notoriamente en los últimos 20 años, las cuales tienden hoy a presentar una mayor masa muscular y un menor contenido de grasa y, como consecuencia, una concentración de PCDDs y PCDFs en el tejido adiposo presumiblemente mayor (Brambilla y col 2008). El modelo desarrollado en el presente estudio considera que la concentración de dioxinas en la grasa equivale a la carga corporal total dividida por la grasa total acumulada, por lo tanto, un menor tenor graso en el organismo implicará que los compuestos se encuentren más concentrados.

Según los resultados obtenidos en este trabajo, las concentraciones de PCDDs, PCDFs y DL-PCBs son mayores a los 120 días que al momento del beneficio (180 días), por esta razón faenar a los animales a edades más tempranas no sería una medida recomendable. Al respecto, Fries (1996) comparó la concentración predicha y observada de heptacloro y oxyclordano, en cerdos contaminados, que comenzaron a ingerir una dieta limpia a los 77 días de edad, observándose una disminución de la concentración de estos compuestos en los animales. El autor explica dicha reducción por la dilución en el pool graso, el cual aumenta a medida que el animal crece y asume que hallazgos similares son esperables con PCDDs, PCDFs y DL-PCBs. Hoogenboom y col (2004) también evidenciaron que los niveles de dichos xenobióticos disminuyen rápidamente en animales en crecimiento.

Los resultados de la contribución de cada una de las categorías de insumos al total de contaminación presente en la grasa de cerdo muestran que las materias primas de origen vegetal son las que más aportan, debido a que se incorporan en un alto porcentaje en las raciones ( $90 \%)$ y el segundo mayor aporte fue el de las materias primas de origen mineral. Aún así, se ha visto que los forrajes son de bajo riesgo, a menos que exista depósito vía aérea de contaminantes al ubicarse en zonas cercanas a sitios de incineración de basura (Rappe y col 1987, Brambilla y col 2004) u otras fuentes de emisión. Por otro lado, el suelo también podría originar la contaminación presente en los insumos de origen vegetal, sin embargo, se ha visto que la absorción de dioxinas desde la tierra es despreciable (Roeder y col 1998) y que la única vía por la cual podrían contaminarse sería por el depósito de polvo en su superficie (European Commission 2000), el cual puede ser lavado posterior a la cosecha, dejando de ser un riesgo

En general, todas las categorías de materias primas del conjunto de datos "EROD/H4IIE" presentaron valores bajos, concentrados a la izquierda de la distribución, pero con la presencia de valores extremos en algunos grupos. La categoría "materias primas de origen mineral" fue la que presentó la media más alta de contaminación (16,21 pg TCDD-EQ/g), seguida por la categoría "mezcla de ácidos grasos" (2,03 pg TCDD-EQ/g). 


\section{RESUMEN}

En el presente estudio se evaluó la contribución de los diversos insumos utilizados en la alimentación de los cerdos, como fuente de contaminación de dioxinas, furanos y bifenilos policlorados similares a dioxinas (DLPCBs) de la carne, considerando las variaciones dietarias durante la recría, crianza y engorda. Las materias primas o insumos fueron separadas en diferentes categorías, elaborándose un conjunto de datos con los resultados de análisis mediante el bioensayo EROD/H4IIE (determinación de la actividad de 7-Etoxiresorufina-O-detilasa en la línea celular de hepatoma de rata H4IIE). Además se establecieron dos dietas tipo utilizadas en Chile, considerando los porcentajes de incorporación de materias primas, los cuales varían a lo largo del ciclo productivo del cerdo. Se realizó un análisis descriptivo de la información contenida en el conjunto de datos, caracterizándose el comportamiento de las observaciones. Se propuso un modelo de transferencia, en que la carga corporal de dioxinas aumenta en forma proporcional al consumo de alimentos contaminados. Como resultados se obtuvo la media de contaminación más alta, expresada como equivalentes tóxicos de 2,3,7,8-tetraclorodibenzo- $p$-dioxina derivados del bioensayo EROD/H4IIE (TCDD-EQ/g), en las materias primas de origen mineral, con $16,21 \mathrm{pg}$ TCDD-EQ/g, seguido por las mezclas de ácidos grasos (2,03 pg TCDD-EQ/g). Las concentraciones medias más bajas de contaminación se presentaron en las premezclas $(0,29 \mathrm{pg}$ TCDD-EQ/g) y los aceites vegetales ( 0,35 pg TCDD-EQ/g). Respecto del modelo de transferencia, al evaluar la contribución de los diferentes insumos al total de contaminación de la ración, las materias primas de origen vegetal fueron las que más aportaron, debido al alto porcentaje en que éstas se incorporan en las dietas tipo. El segundo mayor aporte fue el de las materias primas de origen mineral.

\section{AGRADECIMIENTOS}

Al Columbia Enviromental Research Center (CERC) del US Geological Survey, en especial al Dr. Donald Tillitt, a Diane Nicks y Mike Tanner, a la Asociación Gremial de Productores de Cerdo de Chile A.G, al Fondo de Fomento al Desarrollo Científico y Tecnológico (FONDEF-CONICYT), al Dr. Pedro Cattan y a Hugo Schoffer.

\section{REFERENCIAS}

Brambilla G, G Cherubini, S de Filippis, M Magliuolo, A di Domenico. 2004. Review of aspects pertaining to food contamination by polychlorinated dibenzodioxins, dibenzofurans, and biphenyls at the farm level. Anal Chim Acta 514, 1-7.

Brambilla G, AL Iamiceli, F Ferri, A di Domenico. 2008. Normative and pre-normative aspects for the management of actual and perspective POPs in meat and meat products. Meat Sci 78, 25-33.

European Commission. 2000. Opinion of the Scientific Committee on Animal Nutrition (SCAN) on the dioxin contamination of feedingstuffs and their contribution to the contamination of food of animal origin. Adopted on 06 November 2000. Health \& Consumer Protection Directorate-General. Directorate C - Scientific Opinions. C3 - Management of scientific committees II; scientific co-operation and networks, Brussels, Belgium.

Fries G, G Marrow. 1975. Retention and excretion of 2,3,7,8 tetrachlorodibenzo-p-dioxin by rats. J Agr Food Chem 23, 265-273.

Fries G. 1995. A review of the significance of animal food products as potential pathways of human exposure to dioxins. J Anim Sci 73, 1639-1650.

Fries G. 1996. A model to predict concentration of lipophilic chemicals in growing pigs. Chemosphere 32, 443-451.

Guruge KS, N Seike, N Yamanaka, S Miyazaki. 2005. Polychlorinated dibenzo- $p$-dioxins, -dibenzofurans, and biphenyls in domestic animal food stuff and their fat. Chemosphere 58, 883-889.
Hoogenboom LAP, CA Kan, TFH Bovee, G van der Weg, C Onstenk, WA Traag. 2004. Residues of dioxins and PCBs in fat of growing pigs and broiler fed contaminated feed. Chemosphere 57, 32-42.

Huwe J. 2006. Uptake of dioxin-like compounds in growing swine: correlation between experimental and predicted data. Organohalogen Compd 68, 197-200.

Iben C, J Böhm, H Tausch, J Leibetseder, W Luf. 2003. Dioxin residues in the edible tissue of broiler chicken. J Anim Physiol An N 87, 142-148.

Jones D, S Safe, E Morcom, M Holcomb, C Coppock, W Ivie. 1989. Bioavailability of grain and soil-borne tritiated 2,3,7,8-tetrachlorodibenzo-p-dioxin (TCDD) administered to lactating Holstein cows. Chemosphere 18, 1257-1263.

Kennedy SW, SP Jones. 1994. Simultaneous measurement of cytochrome P4501A catalytic activity and total protein concentration with a fluorescence plate reader. Ana Biochem 222, 217-223.

Malisch R. 2000. Increase of the PCDDs/PCDFs-contamination of milk, butter and meat samples by use of contaminated citrus pulp. Chemosphere 40, 1041-1053.

Mason G, T Sawyer, B Keys, S Bandiera, M Romkes, J PiskorskaPliszczynska, B Zmudzka, S Safe. 1985. Polychlorinated dibenzofurans (PCDFs): correlation between in vivo and in vitro structure-activity relationships. Toxicology 37, 1-12.

Pirard C, E de Pauw. 2005. Uptake of polychlorodibenzo-p-dioxins, polychlorodibenzofurans and coplanar polychlorobiphenyls in chickens. Environ Int 31, 585-591.

Pirkle JL, WH Wolfe, DG Patterson, LL Needham, JE Michalk, JC Miner, MR Peterson, DL Phillips. 1989. Estimates of the half-life of 2,3,7,8- tetrachlorodibenzo-p-dioxin in Vietnam veterans of Operation Ranch Hand. J Toxicol Env Health 27, 165-171.

Rappe C, M Nygen, G Lindstrom, HR Buser, O Blaser, C Wuthrich. 1987. Polichlorinated dibenzofurans and dibenzo-p-dioxins and chlorinated contaminants in cow milk from various locations in Switzerland. Environ Sci Technol 21, 964-970.

Roeder RA, MJ Garber, GT Schelling. 1998. Assessment of dioxins in foods from animal origins. J Anim Sci 76, 142-151.

Samsing F. 2010. Análisis de riesgo de la contaminación con dioxinas, furanos y DL-PCBs en carnes de cerdo, por medio de las materias primas que conforman su alimentación. Proyecto de Título para optar al grado de Licenciado en Medicina Veterinaria y al Título de Médico Veterinario. Universidad Mayor, Santiago, Chile.

Schecter A, L Birnbaumb, JJ Ryan, JD Constable. 2006. Dioxins: An overview. Environ Res 101, 419-428.

Schoffer JT, C Bustos-López, Sotomayor, CA Mattar, A González, C Robles, F Samsing, O Acevedo y CE Valdovinos. 2011. Aplicación del bioensayo EROD-H4IIE para la determinación de dioxinas en carnes de pollos broiler: Un estudio de equivalencia con la cromatografía de gases de alta resolución acoplada a espectrometría de masas de alta resolución. Arch Med Vet 43, 259-266.

Spitaler M, C Iben, H Tausch. 2004. Dioxin residues in the edible tissue of finishing pigs after dioxin feeding. J Anim Physiol An $N 89,65-71$.

Svenson BG, A Nilsson, M Hansson, C Rappe, B Akesson, S Skerfving. 1991. Exposure to dioxins and dibenzofurans trough the consumption of fish. N Engl J Med 324, 8-12.

Tillitt DE, JP Giesy, GT Ankley. 1991. Characterization of the H4IIE rat hepatoma cell bioassay as a tool for assessing toxic potency of planar halogenated hydrocarbons in environmental samples. Environ Sci Technol 25, 87-92.

Tillitt DE, TJ Kubiak, GT Ankley, JP Giesy. 1993. Dioxin-like toxic potency in Forster's Tern eggs from Green Bay, Lake Michigan, North America. Chemosphere 26, 2079-2084.

Unión Europea. 2006. Directiva 2006/13/CE de la Comisión de 3 de febrero de 2006, por la que se modifican los Anexos I y II de la Directiva 2002/32/CE del Parlamento Europeo y del Consejo, sobre 
sustancias indeseables en la alimentación animal, en lo referente a las dioxinas y PCB similares a las dioxinas. L 32/44.

Valdovinos C. 2009. Dioxinas y furanos en Chile: Riesgos potenciales de contaminación en la producción avícola y porcina. Monografía.

Programa de Doctorado en Ciencias Silvoagropecuarias y Veterinarias, Facultad de Ciencias Veterinarias y Pecuarias, Universidad de Chile, Santiago, Chile.
Van Eijkeren JCH, MJ Zeilmaker, CA Kan, WA Traag, LAP Hoogenboom. 2006, A toxicokinetic model for the carry-over of dioxins and PCBs from feed and soil to eggs. Food Addit Contam 23, 509-517.

Whyte JJ, CJ Schmitt, DE Tillitt. 2004. The H4IIE Cell Bioassay as an Indicator of Dioxin-like Chemicals in Wildlife and the Environment. Crit Rev Toxicol 34, 1-83. 